\title{
Effect of Curvature-Dependent Surface Elasticity on the Flexural Properties of Nanowire
}

\author{
Mengjun Wu, ${ }^{1}$ Quan Yuan $\mathbb{D D}^{2}{ }^{2}$ Honglin Li $\mathbb{D},{ }^{3}$ Bin Wu $\mathbb{D},{ }^{4}$ Lin Fang, ${ }^{1}$ and Mengyang Huang ${ }^{2}$ \\ ${ }^{1}$ China Merchants Chongqing Communications Research \& Design Institute Co., Ltd., Chongqing 400067, China \\ ${ }^{2}$ School of Civil Engineering, Architecture and Environment, Xihua University, Hongguang Town, Chengdu 610039, China \\ ${ }^{3}$ China Railway Constructionbridge Engineering Bureau Group 3rd Engineering Co., Shenyang 110043, China \\ ${ }^{4}$ Sichuan Yakang Expressway Co., Ltd., Ya'an 625000, China \\ Correspondence should be addressed to Quan Yuan; yuanqxh@163.com
}

Received 28 May 2021; Accepted 14 July 2021; Published 27 July 2021

Academic Editor: Zheng-zheng Wang

Copyright ( $\odot 2021$ Mengjun Wu et al. This is an open access article distributed under the Creative Commons Attribution License, which permits unrestricted use, distribution, and reproduction in any medium, provided the original work is properly cited.

\begin{abstract}
Surface elasticity and residual stress strongly influence the flexural properties of nanowire due to the excessively large ratio of surface area to volume. In this work, we adopt linearized surface elasticity theory, which was proposed by Chhapadia et al., to capture the influence of surface curvature on the flexural rigidity of nanowire with rectangular cross section. Additionally, we have tried to study the bending deformation of circular nanowire. All stresses and strains are measured relative to the relaxed state in which the difference in surface residual stress between the upper and lower faces of rectangular nanowire with no external load induces additional bending. The bending curvature of nanowire in the reference and relaxed states is obtained. We find that flexural rigidity is composed of three parts. The first term is defined by the precept of continuum mechanics, and the last two terms are defined by surface elasticity. The normalized curvature increases with the decrease in height, thereby stiffening the nanowire. We also find that not only sizes but also surface curvature induced by surface residual stress influence the bending rigidity of nanowire.
\end{abstract}

\section{Introduction}

The effect of surface/interface elasticity on the mechanical properties of one-dimensional nanostructures, particularly those of nanowires, has attracted widespread interest $[1,2]$. The equilibrium position and free energy of surface/interface atoms are different from those of internal atoms, and the differences should be considered in predicting the size-dependent elastic properties of nanowire due to the large ratio of surface area to volume. The extensional and flexural properties are strongly affected by the surface characteristics. Three methods have been used to reveal the surface effects. Continuum mechanics formulation provides a global expression for the combination of surface elasticity and bulk deformation, and the defined surface parameters are determined by atomistic calculation or experiments [3].

Gurtin and Ian Murdoch (GM) were the first to establish rigorous mechanics to model the surface elasticity [4]. Miller and Shenoy studied the size-dependent effective stiffness properties of nanosized bar and beams using a core-shell model [5]. Zhang et al. estimated the effect of surface stress on the effective elastic modulus and asymmetric yield strength of nanowire [6]. Wang and Feng presented a theoretical model for investigating the effect of surface elasticity and residual surface tension on the natural frequency of nanobeam [7]. Xu et al. improved a core-shell model composed of a core and a surface shell layer with constant thickness to predict the effective elastic modulus of nanowire under tension and bending [8]. However, the intrinsic flexural resistance of the surface is ignored in the aforementioned model. The surface energy of nanowire should depend not only on the surface strain but also on the surface curvature. Steignmann and Ogden (SO) established a more general model for surface energy; this model depends on surface curvature in addition to in-plane stretch and shear [9]. Chhapadia et al. provided a simplified and 
linearized version of the model to study the influences of curvature dependence of surface energy on the effective elastic modulus of a thin cantilever beam under pure bending [10]. Gao et al. proposed a curvature-dependent interface energy function to study the nature of the interface stress and bending moment in a nanostructure [11].

The two most studied types of cross section of nanowire are the rectangular and circular sections. Circular nanowire with no external load may also present surface residual and couple stresses due to the initial curvature. The extensional properties of circular nanowire have been discussed in detail in $[12,13]$. Rectangular nanowire represents different mechanisms on the surface from the circular nanowire. The nonuniform surface residual stress may induce the bending of nanowire, which corresponds to a relaxed state. Plane upper and lower surfaces will have a relaxation bending curvature that occupies a part of the surface energy. In this work, simple beam theory and the GM and SO models are adopted to predict the flexural properties of rectangular nanowire.

\section{Model Analysis}

In this section, the reference state of nanowire shown in Figure 1 is considered. The nanowire has four faces, namely, the upper surface, the lower surface, and two profile surfaces. The nanowire has thickness $h$, width $b$, and length $l$. $h$ defines the size of nanowire. The definition of surface parameters depends on the constitutive relationship of nanowire, which is obscure in surface/interface mechanics. The hyperelastic model is the most commonly used constitutive model in which the surface energy density can be expressed by a function of the invariants of surface strain and relative curvature tensors $[9,12]$. The derived relationship among surface stress, surface strain, and curvature denotes the usual nonlinear elasticity. In particular, a linearized curvaturedependent surface elasticity can be obtained for the infinitesimal deformation of nanowire [9]. Miller and Shenoy adopted Timoshenko's symmetric bending theory to obtain the surface stress difference between the upper and lower surfaces [5]. Chhapadia et al. provided a correction of curvature dependence of surface to Stoney's formula [10]. The constructions are defined in the reference or undeformed state of nanowire. However, the difference in surface residual stress between the upper and lower faces of nanowire with no external load may induce additional bending, which corresponds to a relaxation state. All stresses and strains in the nanowire will be measured relative to this state.

Following Chhapadia's formulation, only surface tension and compression along the axial direction on the upper and lower surfaces are considered, and they are assumed to be uniform along the width direction. The surface stresses can be expressed by

$$
\left\{\begin{array}{l}
\tau_{\mathrm{su}}=\tau_{\mathrm{su} 0}+b_{\mathrm{su}} \varepsilon_{\mathrm{su}} \\
\tau_{\mathrm{sl}}=\tau_{\mathrm{s} \mathrm{0}}+b_{\mathrm{sl}} \varepsilon_{\mathrm{sl}}
\end{array}\right.
$$

where $\tau_{\text {su } 0}$ and $\tau_{\text {sl0 }}$ denote the surface residual stresses on the upper and lower surfaces, respectively; $\varepsilon_{\mathrm{su}}$ and $\varepsilon_{\mathrm{sl}}$ denote the surface strains; and $b_{\text {su }}$ and $\tau_{\text {slo }}$ are the material constants associated with surface strains. Under a constant bending moment $M$, the axial strain in bulk is given by

$$
\varepsilon_{x}=-\kappa_{y}\left(y-h_{y}\right)
$$

where $\kappa_{y}$ is the bending curvature of nanowire and $h_{y}$ is the height of neutral axis from the lower surface. The surface strains are determined by the axial strain at $y=0$ and $y=h$. Thus, we have

$$
\left\{\begin{array}{l}
\varepsilon_{\mathrm{su}}=-\kappa_{y}\left(h-h_{y}\right), \\
\varepsilon_{\mathrm{su}}=\kappa_{y} h_{y}
\end{array}\right.
$$

We assume that the residual stain in bulk in the relaxed state is linearly distributed along the height direction. Thus, we have

$$
\varepsilon_{x 0}=-\kappa_{y 0}\left(y-h_{y 0}\right),
$$

where $\kappa_{y 0}$ is the relaxation curvature of nanowire and $h_{y 0}$ is the height of neutral axis in the reference state. Therefore, the stress in the bulk is given by

$$
\sigma_{x}=E\left(\varepsilon_{x}-\varepsilon_{x 0}\right),
$$

where $E$ is the elastic modulus of bulk material. For the upper and lower surface layers, the surface couple is given by

$$
\left\{\begin{array}{l}
m_{\mathrm{su}}=C_{u}\left(\kappa_{y}-\kappa_{y 0}\right) \\
m_{\mathrm{sl}}=C_{l}\left(\kappa_{y}-\kappa_{y 0}\right)
\end{array}\right.
$$

where $C_{u}$ and $C_{l}$ are the SO constants. We can establish the equilibrium equations by using the internal force balance of simple beam with the effect of surface stress. Surface stress, surface couple stress, and stress in bulk balance the applied moment $M$, and the integration over the cross-sectional area yields

$$
\int_{0}^{h} \sigma_{x}(h-y) b \mathrm{~d} y+h b \tau_{\mathrm{sl}}+b\left(m_{\mathrm{su}}+m_{\mathrm{sl}}\right)=M .
$$

Thus, we have

$$
\kappa_{y}=\frac{M+\left[E b h^{2}\left(3 h_{y 0}-h\right) / 6+b\left(C_{u}+C_{l}\right)\right]-b h \tau_{\mathrm{sl} 0}}{E b h^{2}\left(3 h_{y}-h\right) / 6+b_{\mathrm{sl}} b h h_{y}+b\left(C_{u}+C_{l}\right)} .
$$

We balance the force, that is,

$$
\int_{0}^{h} \sigma_{x} \mathrm{~d} y+\tau_{\text {su }}+\tau_{\text {sl }}=0
$$

which gives us

$$
h_{y}=\frac{\kappa_{y} h\left(E h / 2+b_{\mathrm{su}}\right)+\kappa_{y 0} E h\left(h_{y 0}-h / 2\right)-\left(\tau_{\mathrm{s} 10}+\tau_{\mathrm{su} 0}\right)}{\kappa_{y}\left(E h+b_{\mathrm{su}}+b_{\mathrm{sl}}\right)} .
$$

If the surface stress emerges on the front and back surfaces by repeating the analysis of the preceding few pages, then the strains and stresses on the surfaces and in the bulk can be obtained. We now consider the curvature of nanowire 


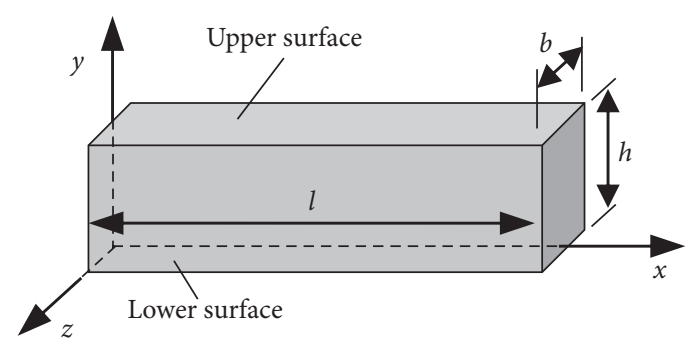

(a)

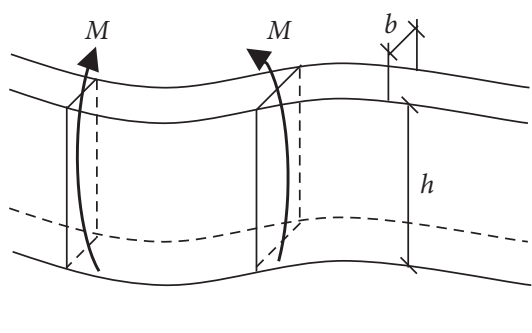

(b)

FIgURE 1: Configuration of nanowire. (a) Reference configuration. (b) Relaxation configuration.

in the relaxed state. We can use equations (8) and (10) to obtain the curvature at which the bending moment vanishes. We let $M=0$ and $\kappa_{y}=0$. Thus, we have

$$
h_{y 0}=\frac{\tau_{\mathrm{su} 0}+\tau_{\mathrm{sl} 0}}{E \kappa_{y 0}}+\frac{h}{2}
$$

where $h_{y 0}$ is the height of neutral axis and is measured in the reference state. By substituting equation (11) into equation (8), we obtain

$$
\kappa_{y 0}=\frac{h \tau_{\mathrm{s} 10}}{E h^{2}\left(3 h_{y 0}-h\right) / 6+C_{u}+C_{l}}=\frac{6 h \Delta \tau_{s 0}}{E h^{3}+12\left(C_{u}+C_{l}\right)},
$$

where $\Delta \tau_{s 0}=\tau_{\mathrm{sl} 0}-\tau_{\mathrm{su} 0}$. The above equation gives the same expression as Chhapadia's.

But for circular nanowire, the surface property is more complicated than that of rectangular nanowire. There is no two-dimensional periodicity on the cylindrical surface. The surface presents strong anisotropy. It is difficult to determine the surface elastic constants. The deformation in bulk induced by surface residual stress is still unclear. To qualitatively analyze the flexural properties of circular nanowire, we consider an isotropic surface without residual stress. For simplicity, the $x$ axis is placed at the axial line of nanowire. Essentially repeating the analysis of the rectangular nanowire, the stress in the bulk is given by

$$
\sigma_{x}=-E \kappa_{y} y .
$$

The above equation is derived by letting $h_{y}=d / 2$ and $\kappa_{y 0}=0$ in equation (5). The surface strain is

$$
\varepsilon_{s}=-\frac{\kappa_{y} d \sin \varphi}{2}
$$

where $\varphi$ is the polar angle on the cross section of nanowire. There are two curvatures on the cylindrical surface after the deformation of nanowire. One is $d / 2$ which is independent of the deformation. The other curvature is $\kappa_{y}$. The surface stress and surface moment stress are

$$
\begin{aligned}
\tau_{s} & =C_{0} \varepsilon_{s}, \\
m_{s} & =C_{1} \kappa_{s} .
\end{aligned}
$$

Similarly balancing the moment, we have

$$
\kappa_{y}=\frac{M}{E I_{z}+C_{0} I_{s}+C_{1} S},
$$

where $I_{z}=\pi d^{4} / 64$ is the moment of inertia of the beam cross section, $I_{s}=\pi d^{3} / 8$ is the perimeter moment of inertia, and $S=\pi d$. The effective bending rigidity is defined as

$$
E^{*} I_{z}=E I_{z}+C_{0} I_{s}+C_{1} S,
$$

where $E^{*}$ is the effective elastic modulus. Equation (16) can be rewritten as

$$
\kappa_{y}=\frac{M}{E^{*} I_{z}}=\frac{M}{E I_{z}\left(1+8 C_{0} / d E+64 C_{1} / d^{3} E\right)} .
$$

We see that the surface elasticity has a definite influence on the bending rigidity of circular nanowire.

\section{Results and Discussion}

We compare the curvature changes in the relaxed and pure bending moment loading states to explain the influence of curvature-dependent surface elasticity on the flexural properties of nanowire clearly. Chhapadia et al. [10] carried out an atomistic simulation of a silver nanowire with a thickness ranging from $1.6 \mathrm{~nm}$ to $6 \mathrm{~nm}$. They found that $C_{u}=C_{l}=C, \quad b_{\mathrm{su}}=b_{\mathrm{sl}}=b, \quad C=-42.3155 \mathrm{eV}, \quad$ and $b=-0.37938 \mathrm{eV} / \AA^{2}$ for the $\langle 100\rangle$ axially oriented nanowire, and $C=114.1895 \mathrm{eV}$ and $b=2.5227 \mathrm{eV} / \AA^{2}$ for the $\langle 110\rangle$ axially oriented nanowire. The effective elastic constants for the $\langle 100\rangle$ surface orientations are negative, thereby softening the nanowire. Only the surface couple stresses on the upper and lower surfaces are considered in this discussion. The configuration of the nanowire is set to be $\langle 110\rangle$ axially, and the positive constants are adopted here.

Figure 2 presents the normalized curvature of nanowire $\left(\kappa_{y 0} /\left(6 \Delta \tau_{s 0} / E h^{2}\right)\right.$ versus the height in the relaxed state. The normalized curvature without SO correction $(C=0)$ does not vary with the change in height, whereas the normalized curvature with SO correction decreases with the decrease in height. This condition implies that the relative stiffness of nanowire increases with the increase in height. The positive SO constant $C$ induces additional surface energy, thereby 


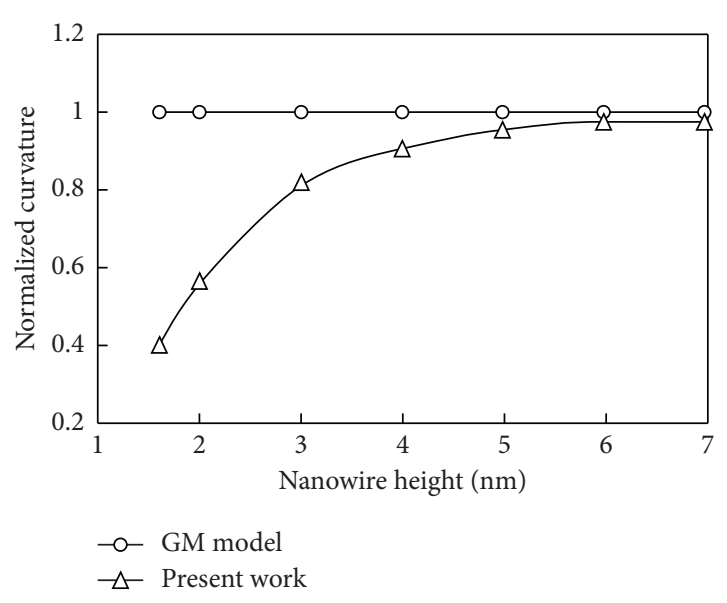

FIGURE 2: Curvature of $\langle 110\rangle$ axially oriented silver nanowire in reference state.

stiffening the nanowire. Thus, the negative $\mathrm{SO}$ constant has a softening influence on the $\langle 100\rangle$ axially oriented nanowire. Notably, the difference in surface residual stress on the upper and lower surfaces induces the relaxation curvature of nanowire. If $\Delta \tau_{s 0}=0$, that is, $\tau_{\text {su } 0}=\tau_{\text {sl0 }}$, then equation (12) shows that $\kappa_{y 0}=0$, and no bending deformation emerges in the relaxed state. The height of neutral axis $h_{y 0}$ has no meaning in such a case. If $\Delta \tau_{s 0}=2 \tau_{\mathrm{sl} 0}$, that is, $\tau_{\mathrm{su} 0}+\tau_{\mathrm{sl} 0}=0$, then $h_{y 0}=h / 2$. In pure bending loaded state, $h_{y}=h / 2$. By substituting equation (11) into equation (10), we can see that the height of neutral axis $h_{y}$ does not affect the surface residual stress, the height of neutral axis, and the curvature in relaxed state. Therefore, the choice of reference or relaxed configuration does not influence the bending deformation of nanowire.

Equation (8) indicates that flexural rigidity is composed of three parts. The first term is defined by the precept of continuum mechanics, and the last two terms are defined by surface elasticity. We can also conjecture that the positive GM and $\mathrm{SO}$ constants will increase the flexural rigidity, whereas the negative ones will decrease the rigidity of nanowire. Figure 3 presents the normalized curvature $\left(12 \kappa_{y} / E b h^{3}\right)$ versus the height of the $\left\langle\begin{array}{llll}1 & 1 & 0\end{array}\right\rangle$ axially oriented nanowire. The figure shows that the normalized curvature also increases with the decrease in height, thereby stiffening the nanowire. For a $2 \mathrm{~nm}$-high nanowire, its curvature changes by $8.2 \%$ when SO and GM corrections are applied. Notably, the bending curvature $\left(\kappa_{y}\right)$ is independent of the surface residual stress. It is the relaxation curvature of nanowire $\left(\kappa_{y_{0}}\right)$ that is influenced by the surface residual stress. For the isotropic circular nanowire without surface residual stress, the components of rigidity are the same as the rectangular nanowire. We also find the stiffening effect for the positive SO and GM constants and the reverse for negative constants.

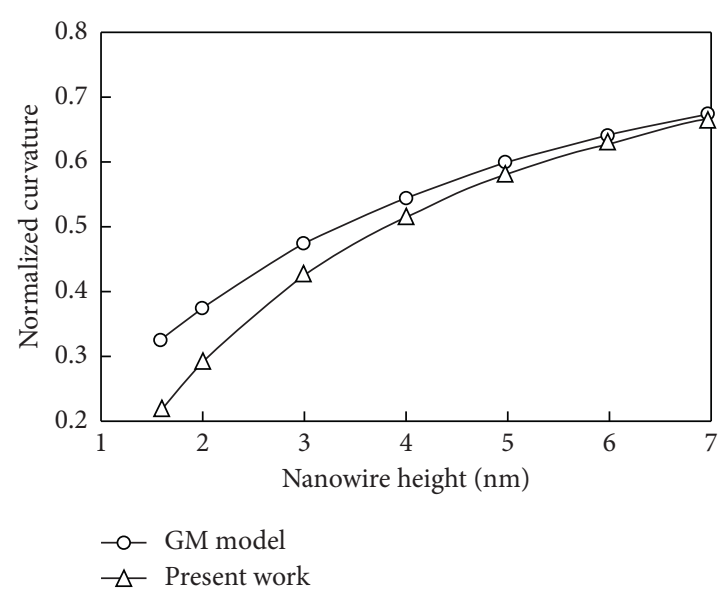

FIGURE 3: Curvature of $\langle 110\rangle$ axially oriented silver nanowire in relaxed state.

\section{Conclusions}

In this work, simple beam theory and curvature-dependent surface elasticity are adopted to capture the flexural properties of nanowire. Surface tension is depicted by the GM model, and surface couple stress is depicted by the SO model. Following the work of Chhapadia et al., we divide the bending deformation of nanowire into the reference and relaxed states. We obtain the expressions of bending curvature and height of neutral axis by using the internal force balance of simple beam with the effect of surface stress. In the relaxed state, the relaxation bending curvature caused by the surface residual stress difference between the upper and lower faces relates to the height of nanowire and the SO constant. The bending rigidity increases with the decrease in the height of nanowire and the positive SO constant. In particular, the height of neutral axis will be half of the height of nanowire if the surface residual stress on the upper and lower surfaces is asymmetrically distributed along the axial direction. In the pure bending moment state, the bending curvature relates to the height of nanowire and the GM and SO constants. The bending rigidity also increases with the decrease in the height of nanowire and the positive GM and SO constants, thereby stiffening the nanowire. Therefore, not only sizes and elastic modulus of bulk material but also relaxation surface curvature induced by surface residual stress influence the bending rigidity of nanowire.

\section{Data Availability}

The cited data about surface elastic constants of silver nanowire used to support the findings of this study are included within the referenced article. These data were used to verify our theoretical prediction. 


\section{Conflicts of Interest}

The authors declare that they have no conflicts of interest.

\section{Acknowledgments}

The authors gratefully acknowledge the financial support provided by the National Engineering Laboratory for Highway Tunnel Construction Technology (grant no. NELFHT201702) and Key Research Project of Xihua University (grant no. z1320608).

\section{References}

[1] D. Appell, "Wired for success," Nature, vol. 419, no. 6907, pp. 553-555, 2002.

[2] E. Gil-Santos, D. Ramos, J. Martínez et al., "Nanomechanical mass sensing and stiffness spectrometry based on two-dimensional vibrations of resonant nanowires," Nature Nanotechnology, vol. 5, no. 9, pp. 641-645, 2010.

[3] S. Wang, Z. Shan, and H. Huang, "The mechanical properties of nanowires," Advanced Science, vol. 4, no. 4, Article ID 1600332, 2017.

[4] M. E. Gurtin and A. Ian Murdoch, "A continuum theory of elastic material surfaces," Archive for Rational Mechanics and Analysis, vol. 57, no. 4, pp. 291-323, 1975.

[5] R. E. Miller and V. B. Shenoy, "Size-dependent elastic properties of nanosized structural elements," Nanotechnology, vol. 11, no. 3, pp. 139-147, 2000.

[6] W. Zhang, T. Wang, and X. Chen, "Effect of surface stress on the asymmetric yield strength of nanowires," Journal of Applied Physics, vol. 103, no. 12, Article ID 123527, 2008.

[7] G.-F. Wang and X.-Q. Feng, "Effects of surface elasticity and residual surface tension on the natural frequency of microbeams," Applied Physics Letters, vol. 90, no. 23, Article ID 231904, 2007.

[8] F. Xu, Q. Qin, A. Mishra, Y. Gu, and Y. Zhu, "Mechanical properties of $\mathrm{ZnO}$ nanowires under different loading modes," Nano Research, vol. 3, no. 4, pp. 271-280, 2010.

[9] D. J. Steigmann and R. W. Ogden, "Elastic surface substrate interactions," Proceedings of the Royal Society of London, vol. 455, no. 1982, pp. 437-474, 1999.

[10] P. Chhapadia, P. Mohammadi, and P. Sharma, "Curvaturedependent surface energy and implications for nanostructures," Journal of the Mechanics and Physics of Solids, vol. 59, no. 10, pp. 2103-2115, 2011.

[11] X. Gao, Z. Huang, J. Qu, and D. Fang, "A curvature-dependent interfacial energy-based interface stress theory and its applications to nano-structured materials: (I) general theory," Journal of the Mechanics and Physics of Solids, vol. 66, pp. 59-77, 2014.

[12] P. Gupta and A. Kumar, "Effect of surface elasticity on extensional and torsional stiffnesses of isotropic circular nanorods," Mathematics and Mechanics of Solids, vol. 24, no. 6, pp. 1613-1629, 2019.

[13] H. Y. Yao, G. H. Yun, N. Bai, and J. G. Li, "Surface elasticity effect on the size-dependent elastic property of nanowires," Journal of Applied Physics, vol. 111, no. 8, Article ID 083506, 2012. 